\title{
Dynamic Virtual Teams in Project Management
}

\author{
Esra'a Alhasanat, Mohammed Alawadi and Mustafa Hammad \\ Department of Information Technology, Mu'tah University, AL Karak, Jordan \\ hammad@mutah.edu.jo
}

\begin{abstract}
Nowadays, with the increasingly important role of technology and internet in our world, it has become not only possible, but necessary for organizations that work globally to cooperate in teams that are spread out worldwide. These "Virtual Teams" (VTs) are imperative to the effective functioning of globalized organizations. Therefore, to improve the work of such organizations, it is necessary to study and understand the dynamics of information sharing and international cooperation among these geographically separated $V T s$. There are many researchers who talk about VTs, but they do not go into deep details about the importance of leadership, conflicting management, and communication tools. We start this research from the points of view of the people who play different roles in $V T s$. In order to get more realistic results, all the given suggestions and solutions must be more practical and meaningful. Our paper focuses on the factors that should be taken into consideration to get a dynamic VT. As a result, we come up with some advices and recommendations to lead VTs to become as successful as possible based on using communication tools, conflicting management, and leadership skills.
\end{abstract}

Keywords: virtual team, conflict management, trust, communication tools

\section{Introduction}

In spite of the fast development of technology, and the increasing of numbers and types of communication tools, the virtual world still suffers from many problems. These problems affect negatively on the project's environment that depends on VTs. A VT is defined as a group of people who geographically live far away from each other [1]. All VTs, sometimes called Global Virtual Teams (GVTs) or even multi-cultural virtual teams, share several characteristics. They operate by electronic communication, are nonpermanent, and are diverse with respect to the cultures and geographical locations of their members. However, there is great variation among VTs in terms of the characteristics of space, time, and configuration [2] .For this reason; many issues arise in working within a VT framework. Many of these issues are dealt with by Rutkowski et al. with special attention given to the dynamics of information sharing and the ability of management to mitigate problems. Griffith et al. suggests proactive structuration as an effective approach to VTs, while Chidambaram [3] discusses the importance of adhering to milestones on a timeline as a way to encourage team members and to draw from the benefits of a diverse team. In such projects, where members are geographically separated, VTs play the main role to achieve their objectives successfully, and to have an effective VT.

Communication is considered one of the most important elements for the VT's success. We can define communication as a complementary element to any team to lead it to success, and it has a key role in increasing the trust level among members in the team. In the absence of the suitable communicating tools, [4] argues that it is useless to create such teams. So, we must benefit from those tools in the best way. Trust [5] is another major consideration to successful VTs. Trust in VTs is defined as the level of confidence among the team members. 
We also consider leadership as an important factor in the successful VT [6]. The team's leader has the responsibility to present timely feedback and reflection, and supply members with resources, information sharing, and contacts .Therefore, every team needs good leadership to organize its tasks and activities on one hand, and to solve its conflicts on the other hand. In traditional teams, the team needs a responsible person to make some decisions and plan the tasks.

Managing conflicts [7] and solving problems plays a main and significant role in any VT, so if these problems and conflicts are not solved and controlled, the project will be stopped and the team will waste its time and effort. So, start by managing the conflicts [8] to avoid most of such problems. By using this technique, time and effort can be reduced.

This paper proposes the successful keys for VTs from the member's perspective. The second section of the paper shows the related work. We briefly describe the methodology in the Section 3. In Section 4, we analyze the findings to get the results follows by tips and advice to build a dynamic VT. Finally, the conclusion summarizes the findings and results.

There are many of characteristics like communication tools, conflict management, good planning wisdom leadership and trust building; they show the most important points that must be taken into consideration to build a successful team.

\section{Related Work}

There are many studies that have been done to improve the concepts of VTs in project management. In [9] they talk about the crucial technological subjects that influence the performance of virtual project teams. Their findings support the following four guidelines. First, establish the trust in the team through using communication technology. Second, use technology to monitor team progress. Third, enhance the visibility of virtual members within the team and outside the organization. Finally, benefit from the team for individual members through VT settings.

In [10] they have examined the performance of globally distributed VTs through an exploratory experimental study. They find a strong relation between communication effectiveness of the team and the trust between the team members. In [11] study the supporting virtual software project on the web. They experience the modeling and environment of software process to find the major challenges concerning virtual software projects. Then, they have found several separated parties could be supported in software process.

The aim of the researchers in this paper is to examine the effective project management of virtual software teams. They have come up with six factors that need to be addressed. These are organizational structure, risk management, infrastructure, process, conflict management, and team structure. Thus, it requires a special effort from all participants [7].

According to [6], using different skills of project teams such as knowledge, cultures, and perspectives has positive influences on team outcomes. In his paper, he tries to find an answer to this question: To what extent can project management methods and tools benefit in VT to minimize its challenges?

In [8] researchers are inconclusive about the role of conflict within VTs. They argue and study the complex relationship between structural stability, conflict, and VT performance within a project. This study provides a developmental view of the emergence and impact of structural stability within VTs, as well as greater understanding of the contribution of conflict to VT performance.

In a survey study [12] about the major risks in virtual projects of the software industry, they explained that using interact risk identification and assessment factors in an actual business organization concludes in three major risks: communication risk, schedule and cultural risks. They concentrate on the concept of virtual project management. 
In this paper the researchers try to solve the problem of finding the vague factors which influence the effectiveness of VTs for new product development. To find these factors they use a field survey and literature review. They study the knowledge workers in one hand and the technology on the other hand $[4,5]$. Use a field survey to make a standard framework design for organizations to help them understand the relationship between trust and knowledge in global VTs. Their results help them to understand and assess the impact of this relationship. They also help to test the role of technology in understanding the effectiveness of global VTs and the interplay of diversity [9]. focus on how the global VTs might be more helpful than face-to-face teams in certain contests (that require crossfunctional skilled inputs). Their findings in team relations indicate that cultural and time differences (according to the geographical and temporal-distance features) should be taken in consideration.

\section{Methodology}

After a deep study of the subject of VTs in project management, we designed a questionnaire to collect information about VTs and tools used in various activities, and some of the challenges encountered and suggestions for these problems

The survey is divided into three sections. The first section concerns the nationality of the member and his experiences in VTs. Also, it concerns the members' roles and numbers dealt with. In the second section of the study, we ask some questions about the most important needs in the VTs, the conflicts which could be faced, and some suggestions to solve such issues. Also, some questions are raised about the difference between face-to-face teams and VTs in their productivity. The last section of the research is concerned with the technological tools that are used in VTs, which tool is better, and the characteristics of each used tool.

The sample was gathered in many ways, such as publishing the questionnaire by E-mail [13], got some data from a conference that was held at the university. After gathering the sample of the target group (who worked in VTs), we analyzed the data to get the results of the study, that are mentioned in the next section of the paper.

\section{Data Analysis}

The fifty-two respondents were chosen from eleven countries, with different cultures, traditional and different intellectual perspective. These countries are Jordan(20), Pakistan(10), Algeria(8), Yemen(3), USA(2), Malaysia(2), Egypt(2), Australia(1), British(1), Czech(1) and Japan(1), ranged from 23 to 55 years old. More than three quarters of the survey participants $(77 \%)$ are from the research sector in different areas, followed by participants of the educational sector (63\%). In addition, there are participants from finance, industry, and security sectors. Figure 1 shows the most important needs in VT. 


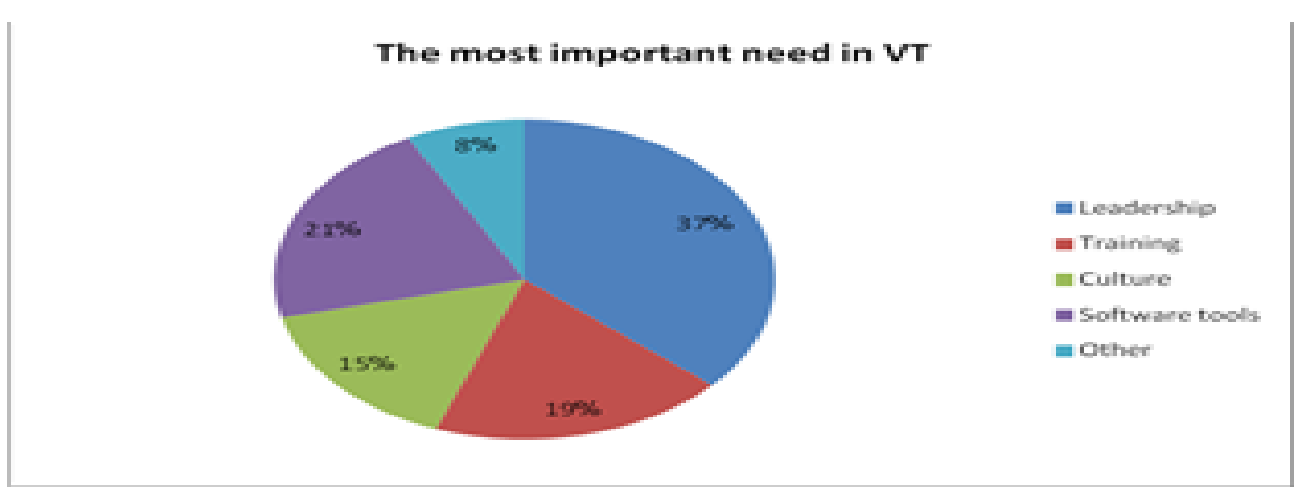

Figure 1. The Most Important Needs in VT

From Figure 1, we can note that the top need in VT is leadership with a percent of (37\%), followed by software tools and training with almost equal proportions (21\% and $19 \%)$. (15\%) of the respondents said that Culture is an important need in VT.

When people were asked more about productive team, virtual or face-to-face, their answers were different. Those people answered this question "Do you feel that your VT meets its objectives as a face-to-face team?" With different answers. Results were as follow: $12 \%$ of people of the sample prefer to work face to face, because they can make easy observation on work, so the group team will be more awareness and they can instantly discuss conflict and solve any problem with each other, in additional more confidence. However, 33\% prefer using VT more than face-to-face; it helps working together along far distance. This ability advantages allow organizations to increase their flexibility and sensibility. $41 \%$ thought that using the two techniques depends on the project itself, because each project has different criteria such as reliability, efficiency, confidentiality and etc. As shown in the Table 1.

Table 1. Responses about More Productive Team

\begin{tabular}{|l|c|c|}
\hline \multicolumn{1}{|c|}{ Question } & Response & Percent \\
\hline More than face-to-face. Why? & $6 *$ & $12 \%$ \\
\hline Same as face-to-face. Why? & $7 * *$ & $14 \%$ \\
\hline Less than face-to-face. Why? & $17^{* * *}$ & $33 \%$ \\
\hline Depends on the project itself. & 21 & $41 \%$ \\
\hline
\end{tabular}

*Those whose responses are more than face-to-face justify their answer as they can make decisions and have collaborated with less effort, less time and, of course, less cost, also they can easily understand and negotiate the given issues to be handled.

**Those who answer that VT is similar to the face-to-face team, because using communication tools, like Skype, allows them to do a face-to-face discussion which gives them the same advantages of the face-to-face meeting, also, because no hardware tools or equipment are needed at these meetings.

***The last group says face-to-face teams achieve their goals better than VTs, since the members in VTs are not serious enough, and sometime they misunderstand each other, or there are management problems. 
Results show that project teams still prefer to work through traditional meetings, i.e., VTs still need more training and improvement tips to increase the confidence of VT's idea. In spite of the technological environment, our study shows that the most commonly used tool is E-mail.

Another question is about the way that the members use to avoid conflicts, and the responses are shown in the following chart in Figure 2.

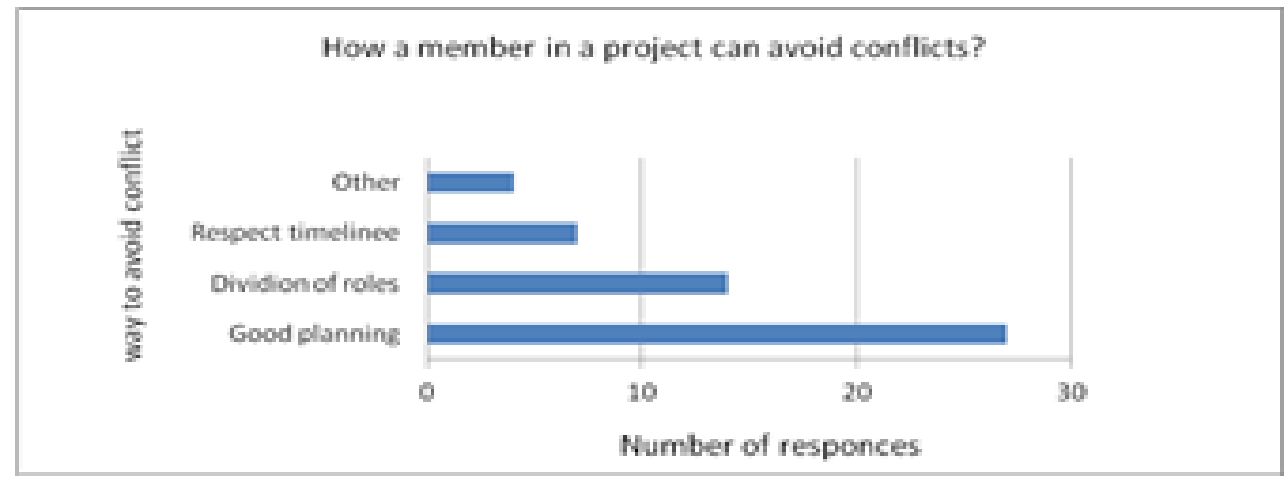

Figure 2. How a Member Can Avoid Conflicts?

Most of the participants mention that the success of any VT depends mainly on good planning, followed by respondents who choose avoiding conflicts by dividing the roles and responsibilities among the members of the team. Respecting time comes in the third place. Others say that you need to use skills to manage your conflicts.

When we ask some participants about the way of solving the conflicts in VTs, they give different opinions. Most of them consider this task as a manager's role, but for others, it should be solved between the members of the team.

In their opinion, as the manager is the responsible person in the team, he should start by planning the team in a good way, giving a task to everyone and dividing the roles in a suitable way. In this way, the leader can ensure that each member feels he is an integral part of the group and has a full opportunity to participate to provide a base for education, including the ability to listen, work in groups and solve problems. Then, when there is a problem, the leader should explain it to the other members and try to find a solution without searching who is responsible for the conflict. Some say that the leader should talk to members privately to reach an agreement between them, but at the same time, if they do not find a solution, they must make critical decisions. Others say that members should clarify their ideas with some diplomacy using technology tools, such as Skype calls, e-mails, chatting, or phoning. On the other hand, a small group of the participants has a contrary opinion to the VT's criteria which includes arranging face-to-face meetings.

Other opinions hold the responsibility of solving problems upon the members themselves, not the manager by accepting or refusing the roles from the beginning, discussing the route and the reasons of the problem to find out a solution. However, they all should take respecting the time line in consideration to avoid such conflicts.

When we ask the people about their preferable communicating tool(s) in VT, they response in different ways as shown in Figure 3. 


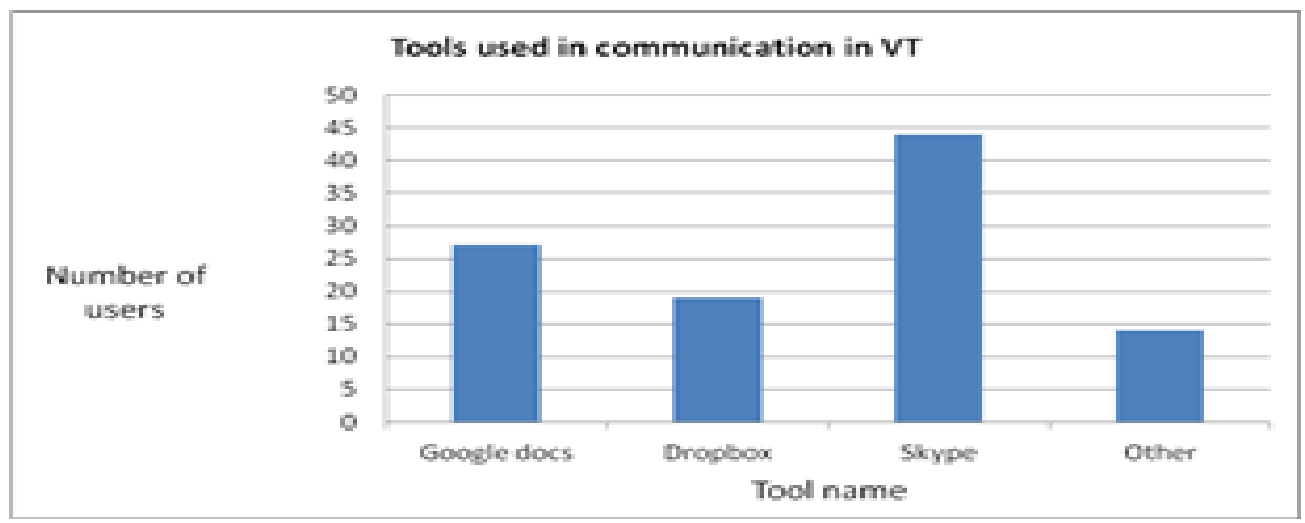

Figure 3. Tools used in Communication in VT

From Figure 3, we can notice that $(85 \%)$ persons prefer Skype calls or chat for these reasons:

-It is similar to face-to-face meetings.

-It is free, easy to use and it supports video, audio, and written chat.

-It helps the members interact with each other and share documents by providing some functionality.

-It helps in brainstorming, and makes decisions quickly while miles apart.

On the other hand, some complain about the need high rate of internet connection, especially for video communication, the lack of reliable interest connection and body language, government can eavesdropping on calls, other disadvantage is when your computer turned off (i.e., electricity problems), you will not be able to receive any call, so when your leadership call you for an urgent situation then you cannot answer him this will effect on the workflow. In addition to differences in time zones problems.

\section{Tips to Help You Build an Effective Virtual Team}

According to our questionnaire that we distributed randomly by using the E-mail and conference, we gathered the answers and analyzed them precisely then we concluded these tips to help people building an effective VT.

1. The team's leader has great responsibilities in managing problems, so choose a successful leader, who leads you to a successful project. A deep study of the characteristics of the leader and training courses via the Internet will be helpful, before proceeding with the project.

2. Managing conflicts is an essential part while developing the plan; this assumption will protect your team from the problems that may encounter leader and members.

3. Choose the suitable technology tool. The development of the technological tools makes it easier for us in doing things. These tools are there to support people in VTs, so there is no need to be without them anymore. These tools include: the internet, video cams, and shared directories. At the same time, however, be sure to choose the suitable tool.

4. Be aware of cultural differences. Of course, when you are a project manager in a VT, you will expect different members from different cultures and traditions. So they will not behave and work in the same way. Make sure you have some appreciation for the differences between the members and try to understand them.

5. Maybe the members in VTs never meet each other face-to-face; this requires considerable effort to build confidence. 
6. All the members of the team should know and understand the whole work. Not only their own role, but also they must share the ideas between each other. Because if they work individually, they will just be individual contributors.

7. Be aware of time differences. Do not forget the differences in time zones. Thus, if it is suitable for you to talk at a certain time, it may be harder for others because of their time zone.

8. Respect the timeline. One of the basic rules that help to improve the VTs is to set the working and meeting hours among the members. Each member should respect this time and meet the milestones on time.

9. Wide communication protects members from some conflicts and misunderstandings, which increases the level of confidence. One of the most irritable factors that affect the team's work is the misunderstanding between the members, since they come from different countries and different cultures. To minimize this problem as much as possible, you, as a leader, help the members communicate and talk freely to protect the team from most of the conflicts and to minimize the cultural gap between them.

10. Assign shorter tasks to members. Do not overload the members with work and tasks. So, to reduce the conflicts and to avoid postponing tasks, give the members short assignments to expect better results on time.

\section{Conclusion}

This paper introduces the effective keys in VTs. The results we have manipulated and analyzed provide a good idea about how to form a successful VT. Our study's findings focus on two main factors. The first one is the leadership role in avoiding conflicts as much as possible. The second one depends on technological tools and their roles in communication, sharing knowledge, managing conflicts, etc. For future researches, we will go into a detailed and comprehensive study for the factors that affect the VTs. We will apply it to a larger sample to design a clear VT's framework.

\section{References}

[1] B. Seerat, M. Samad and M. Abbas, "Software Project Management in Virtual Teams", Science and Information Conference, (2013), pp. 139-143.

[2] A. Martinić, Virtual Teams, (2009).

[3] L. Banica, D. Rosca and C. Stefan, "A Software for Project Management Process", Proceedings of the Romanian Academy, vol. 10, no. 2, (2009), pp. 179-187.

[4] N. A. Ebrahim, S. Ahmed, S. A. Rashid and Z. Taha, "Effective virtual teams for new product development", Scientific Research and Essays, vol. 7, no. 21, (2012), pp. 1971-1985.

[5] P. Pinjani and P. Palvia, "Trust and knowledge sharing in diverse global virtual teams", The International Journal of Information Systems Theories and Applications, (2013), pp. 144-153.

[6] C. Beise, "IT Project Management and Virtual Teams", sSIGMIS CPR '04 Proceedings of the 2004 SIGMIS conference on Computer personnel research, (2004), pp. 129 - 133.

[7] V. Casey and I. Richardson, "Project Management within Virtual Software Teams", Global Software Engineering, ICGSE '06. International Conference on, (2006), pp. 33-42.

[8] P. Weimann, M. Pollock, E. Scott and I. Brown, "Enhancing Team Performance Through Tool Use", How Critical Technology-Related Issues Influence the Performance of Virtual Project Teams, (2013), pp. 332-353.

[9] L. Lee-Kelley and T. Sankey, "Global virtual teams for value creation and project success: A case study", International Journal of Project Management, vol. 26, (2007), pp. 51-62.

[10] V. Sridhar, R. Paul D. Nath and K. Kapur, "Analyzing Factors That Affect Performance Of Global Virtual Teams", (2007), pp. 159-170.

[11] K. Alho and R. Sulonen, "Supporting Virtual Software Projects on the Web", Enabling Technologies: Infrastructure for Collaborative Enterprises, (1998), pp. 10-14.

[12] I. Basharat, T. Nafees and M. Abbas, "Risks factors identification and assessment in virtual projects of software industry: A survey study", Science and Information Conference (SAI), (2013), pp. 176 - 181.

[13] "Qualtrics questionnaire Web Site", (2014) November 4, https://qtrial2014.az1.qualtrics.com/SE/?SID=SV_2iy9yhYnt6t3fq5. 
International Journal of $u-$ and $\mathrm{e}-$ Service, Science and Technology

Vol.8, No. 7 (2015)

\section{Authors}

Esra'a Alhasanat, a researcher in computer science field, (esraahasanat_88@hotmail.com)

Mohammed Alawadi, a researcher in computer science field (mohdahu87@gmail.com)

Mustafa Hammad, Assistant Professor at Mutah University (hammad@mutah.edu.jo) 